\title{
Geometric characterization for affine mappings and Teichmüller mappings
}

\author{
by \\ Zhiguo Chen (Hangzhou)
}

\begin{abstract}
We characterize affine mappings on the unit disk and on rectangles by module conditions. The main result generalizes the classic Schwarz lemma. As an application, we give a sufficient condition for a $K$-quasiconformal mapping on a Riemann surface to be a Teichmüller mapping.
\end{abstract}

1. Preliminaries. Let $\Gamma$ be a family of curves in the plane. Each $\gamma \in \Gamma$ is a countable union of open arcs, closed arcs or closed curves, and every closed subarc is rectifiable. We shall define the extremal length $\lambda(\Gamma)$ of $\Gamma$.

A function $\varrho$, defined on the whole plane, is called admissible if the following conditions are satisfied:

(i) $\varrho$ is a non-negative Borel function,

(ii) $A(\varrho)=\iint_{\mathbb{C}} \varrho^{2} d x d y \neq 0, \infty$.

If such a $\varrho$ is measurable as a function of arc length on $\gamma$, set

$$
L_{\gamma}(\varrho)=\int_{\gamma} \varrho|d z| .
$$

Otherwise, set $L_{\gamma}(\varrho)=\infty$. Define

$$
\begin{aligned}
& L(\varrho)=\inf _{\gamma \in \Gamma} L_{\gamma}(\varrho), \\
& \lambda(\Gamma)=\sup _{\varrho} \frac{L(\varrho)^{2}}{A(\varrho)},
\end{aligned}
$$

where the supremum is taken over all admissible $\varrho$. We write $\Gamma_{1}<\Gamma_{2}$ if for every $\gamma_{2} \in \Gamma_{2}$ there is a $\gamma_{1} \in \Gamma_{1}$ which is a subarc of $\Gamma_{2}$. By the above definitions, the extremal length is monotonic:

2000 Mathematics Subject Classification: 30C62, 30C55, 30F60.

Key words and phrases: quasiconformal mapping, extremal length, Teichmüller mapping.

Research supported by National Science Foundation of China (Grant No. 10101023). 
Proposition 1.1. If $\Gamma_{1}<\Gamma_{2}$, then $\lambda\left(\Gamma_{1}\right) \leq \lambda\left(\Gamma_{2}\right)$.

Let $\Gamma_{1}, \Gamma_{2}$ be two families of curves. Set

$$
\Gamma_{1}+\Gamma_{2}=\left\{\gamma_{1}+\gamma_{2} \mid \gamma_{1} \in \Gamma_{1}, \gamma_{2} \in \Gamma_{2}\right\} .
$$

The extremal length of curve families has the following subadditivity property:

Proposition 1.2. If $\Gamma_{1}$ and $\Gamma_{2}$ lie in mutually disjoint sets, then

$$
\lambda\left(\Gamma_{1}+\Gamma_{2}\right) \geq \lambda\left(\Gamma_{1}\right)+\lambda\left(\Gamma_{2}\right) .
$$

For details about the properties of extremal length we refer to [1].

A quadrilateral consists of a Jordan domain $Q$ and a sequence $z_{1}, z_{2}, z_{3}, z_{4}$ of boundary points of $Q$. The points $z_{i}$ are called the vertices of the quadrilateral, and divide its boundary into four Jordan arcs, called the sides of the quadrilateral. The arcs $z_{1} z_{2}$ and $z_{3} z_{4}$ are called the a-sides and the other two the $b$-sides of $Q$. Let $\Gamma_{a}$ be the family of curves that connect the $a$-sides in $Q$, and $\Gamma_{b}$ the family of curves that connect the $b$-sides in $Q$. Define the module of the quadrilateral $Q\left(z_{1}, z_{2}, z_{3}, z_{4}\right)$ to be $\lambda\left(\Gamma_{a}\right)$, the extremal length of $\Gamma_{a}$. That is,

$$
\bmod Q\left(z_{1}, z_{2}, z_{3}, z_{4}\right)=\sup _{\varrho} \frac{\left(\inf _{\gamma \in \Gamma_{a}} L_{\gamma}(\varrho)\right)^{2}}{A(\varrho)} .
$$

For example, for a rectangle with width $a$ and height $b$, its module is $a / b$.

If $\varrho=1$ in (1.1), then $L_{\gamma}(\varrho)$ is the euclidean length of $\gamma$, simply denoted by $|\gamma|$. We call

$$
s_{a}=s_{a}(Q)=\inf _{\gamma \in \Gamma_{b}}|\gamma|
$$

the distance between the a-sides of $Q$. The distance $s_{b}$ between the b-sides is defined analogously. Let $m(Q)$ be the euclidean area of $Q$. The following Rengel inequality plays an important role in this paper.

Proposition 1.3 ([7]). The module of a quadrilateral $Q$ satisfies the double inequality

$$
\frac{\left(s_{b}(Q)\right)^{2}}{m(Q)} \leq \bmod Q \leq \frac{m(Q)}{\left(s_{a}(Q)\right)^{2}} .
$$

REMARK 1.4. This inequality is usually called Rengel's inequality in the literature. However, it was first given by H. Grötzsch (see [6]).

The theory of quasiconformal mappings is closely related with the study of extremal length. In fact, the geometric definition of a quasiconformal mapping is based on moduli of quadrilaterals, which are represented by extremal length. Precisely, a function $f(z)$, which is a sense-preserving homeomorphism of $\Omega$ onto $\Omega^{\prime}$, is $K$-quasiconformal if for every quadrilateral 
$Q=Q\left(z_{1}, z_{2}, z_{3}, z_{4}\right) \subset \Omega$,

$$
\frac{1}{K} \bmod Q \leq \bmod f(Q) \leq K \bmod Q .
$$

Denote the unit disk by $\Delta$. It follows from the Riemann mapping theorem that every quadrilateral $Q\left(z_{1}, z_{2}, z_{3}, z_{4}\right)$ can be mapped onto a quadrilateral $\Delta\left(\zeta_{1}, \zeta_{2}, \zeta_{3}, \zeta_{4}\right)$ with domain $\Delta$ and vertices $\zeta_{1}, \zeta_{2}, \zeta_{3}, \zeta_{4}$ on $\partial \Delta$. By SchwarzChristoffel,

$$
\Phi(\zeta)=\int^{\zeta} \frac{d \zeta}{\left(\zeta-\zeta_{1}\right)\left(\zeta-\zeta_{2}\right)\left(\zeta-\zeta_{3}\right)\left(\zeta-\zeta_{4}\right)}
$$

conformally maps $\Delta\left(\zeta_{1}, \zeta_{2}, \zeta_{3}, \zeta_{4}\right)$ onto a rectangle. By combining the above mappings, we can map an arbitrary quadrilateral conformally onto a rectangle. Therefore, from conformal invariance of extremal length, the module of any quadrilateral can always be represented by that of its conformally equivalent rectangle.

Define the module of an annulus

$$
A\left(r_{1}, r_{2}\right)=\left\{z\left|r_{1} \leq\right| z \mid \leq r_{2}\right\}
$$

to be the extremal length of the family $\Gamma$ of curves that connect $\left\{z|| z \mid=r_{1}\right\}$ and $\left\{z|| z \mid=r_{2}\right\}$ in $A\left(r_{1}, r_{2}\right)$. Since $\lambda(\Gamma)$ is conformally invariant and every ring domain can be mapped conformally onto an annulus, the number

$$
\bmod A\left(r_{1}, r_{2}\right)=\frac{1}{2 \pi} \log \frac{r_{2}}{r_{1}}
$$

represents the moduli of all ring domains which are conformally equivalent to $A$.

Define the module of a sector

$$
A\left(r_{1}, r_{2} ; \theta_{1}, \theta_{2}\right)=\left\{z\left|r_{1} \leq\right| z \mid \leq r_{2}, \theta_{1} \leq \arg z \leq \theta_{2}\right\}
$$

to be the extremal length of the family $\Gamma$ of curves that connect $\left\{z|| z \mid=r_{1}\right\}$ and $\left\{z|| z \mid=r_{2}\right\}$ in $A\left(r_{1}, r_{2} ; \theta_{1}, \theta_{2}\right)$. Then

$$
\bmod A\left(r_{1}, r_{2} ; \theta_{1}, \theta_{2}\right)=\frac{1}{\theta_{2}-\theta_{1}} \log \frac{r_{2}}{r_{1}} \text {. }
$$

2. Affine mappings on the unit disk $\Delta$. Quasiconformality of a domain is characterized not only by the module of a quadrilateral $Q$ as in inequality (1.5), but also by that of horizontal rectangles. A horizontal rectangle is a quadrilateral whose $a$-sides are parallel to the $x$-axis and $b$-sides are parallel to the $y$-axis. For the relevant results, we refer to [3] and [4]. In this section, we shall prove

THEOREM 2.1. Let $f(z)$ be a sense-preserving homeomorphism of the unit disk $\Delta$ onto itself, with normalization $f(0)=0$. If 


$$
\frac{1}{K} \bmod A \leq \bmod f(A),
$$

where $A$ stands for all $A\left(r_{1}, r_{2}\right)$ and $A\left(r_{1}, r_{2} ; \theta_{1}, \theta_{2}\right)$ as in (1.6) and (1.8), and

$$
\lim _{z \rightarrow 0} \frac{|f(z)|}{|z|^{1 / K}}=1,
$$

then

$$
f(z)=\lambda z|z|^{1 / K-1} \quad(|\lambda|=1) .
$$

In view of Theorem 2.1, we immediately generalize the Schwarz lemma to sense-preserving homeomorphisms.

Corollary 2.2. Assume that $f(z)$ is a sense-preserving homeomorphism of the unit disk $\Delta$ onto itself, with normalization $f(0)=0$. If $f$ satisfies conditions (a) and (b), where $K=1$ in (a), then

$$
f(z)=\lambda z \quad(|\lambda|=1) .
$$

For the proof of the theorem, we need three lemmas.

Lemma 2.3. If $f$ satisfies condition (a) of Theorem 2.1, then it is absolutely continuous on $\{|z|=r\}$.

Proof. Let $A=\left\{z=r e^{i \theta} \mid r_{1}<r<r_{2}, 0 \leq \theta<2 \pi\right\}$ be an annulus in $\Delta$. Set

$$
q(t)=m\left(f\left(A_{t}\right)\right)
$$

where $A_{t}=\left\{z=r e^{i \theta} \mid r_{1}<r<t, 0 \leq \theta<2 \pi\right\}$ and $m\left(f\left(A_{t}\right)\right)$ is the euclidean area of $f\left(A_{t}\right)$. Obviously, $q(t)$ is an increasing function of $t$ and thus has a finite derivative $q^{\prime}(t)$ for all $t, r_{1}<t<r_{2}$, except for a set of zero linear measure. Assume that $q^{\prime}\left(t_{0}\right)$ exists and is finite. We shall prove that $f\left(t_{0} e^{i \theta}\right)$ is absolutely continuous on $[0,2 \pi]$.

We first choose a positive $\delta$ such that $t_{0}+\delta<r_{2}$. Let $\left(\theta_{k}, \theta_{k}^{*}\right), k=1, \ldots, n$, be an arbitrary system of non-intersecting open subintervals of $[0,2 \pi]$. Define a sector (a special quadrilateral) by

$$
G_{k}^{\delta}=\left\{z=r e^{i \theta} \mid t_{0}<r<t_{0}+\delta, \theta_{k}<\theta<\theta_{k}^{*}\right\} .
$$

Then by (1.9),

$$
\bmod G_{k}^{\delta}=\frac{1}{\theta_{k}^{*}-\theta_{k}} \log \frac{t_{0}+\delta}{t_{0}} .
$$

For the module of the image of $G_{k}^{\delta}$, from the right hand side of Rengel's inequality we have

$$
\bmod f\left(G_{k}^{\delta}\right) \leq \frac{m\left(f\left(G_{k}^{\delta}\right)\right)}{\left(d_{k}^{\delta}\right)^{2}}
$$


where $d_{k}^{\delta}$ denotes the euclidean distance between the $a$-sides of $f\left(G_{k}^{\delta}\right)$. These sides converge to the points $f\left(t_{0} e^{i \theta_{k}}\right)$ and $f\left(t_{0} e^{i \theta_{k}^{*}}\right)$ as $\delta \rightarrow 0$, and so

$$
\lim _{\delta \rightarrow 0} d_{k}^{\delta}=\left|f\left(t_{0} e^{i \theta_{k}^{*}}\right)-f\left(t_{0} e^{i \theta_{k}}\right)\right| .
$$

According to condition (a), we have

$$
\frac{1}{K} \bmod G_{k}^{\delta} \leq \bmod f\left(G_{k}^{\delta}\right)
$$

From (2.2), (2.4) and (2.1), we get

$$
\frac{\left(d_{k}^{\delta}\right)^{2}}{m\left(f\left(G_{k}^{\delta}\right)\right)} \leq \frac{K\left(\theta_{k}^{*}-\theta_{k}\right)}{\log \left(1+\delta / t_{0}\right)}, \quad k=1, \ldots, n .
$$

Noticing that $\log \left(1+\delta / t_{0}\right) \geq \delta /\left(2 t_{0}\right)$ for $\delta<t_{0}$ and adding the above inequalities over $k$ we have

$$
\sum_{k=1}^{n} \frac{\left(d_{k}^{\delta}\right)^{2}}{m\left(f\left(G_{k}^{\delta}\right)\right)} \leq 2 K t_{0} \sum_{k=1}^{n} \frac{\theta_{k}^{*}-\theta_{k}}{\delta}
$$

By the Schwarz inequality,

$$
\left(\sum_{k=1}^{n} d_{k}^{\delta}\right)^{2} \leq \sum_{k=1}^{n} \frac{\left(d_{k}^{\delta}\right)^{2}}{m\left(f\left(G_{k}^{\delta}\right)\right)} \sum_{k=1}^{n} m\left(f\left(G_{k}^{\delta}\right)\right)
$$

Observing that

$$
\sum_{k=1}^{n} m\left(f\left(G_{k}^{\delta}\right)\right) \leq q\left(t_{0}+\delta\right)-q\left(t_{0}\right)
$$

we obtain

$$
\left(\sum_{k=1}^{n} d_{k}^{\delta}\right)^{2} \leq 2 K t_{0} \frac{q\left(t_{0}+\delta\right)-q\left(t_{0}\right)}{\delta} \sum_{k=1}^{n}\left|\theta_{k}^{*}-\theta_{k}\right| .
$$

Letting $\delta \rightarrow 0$, from (2.3) and (2.6) we obtain

$$
\left(\sum_{k=1}^{n}\left|f\left(t_{0} e^{i \theta_{k}^{*}}\right)-f\left(t_{0} e^{i \theta_{k}}\right)\right|\right)^{2} \leq 2 K t_{0} q^{\prime}\left(t_{0}\right) \sum_{k=1}^{n}\left|\theta_{k}^{*}-\theta_{k}\right| .
$$

This completes the proof of the lemma.

Define

$$
R(r)=\{z|r \leq| z \mid \leq 1\} .
$$

There exists a conformal maping $\Phi_{r}$ such that

$$
\Phi_{r} \circ f(R(r))=\{\zeta|\phi(r) \leq| \zeta \mid \leq 1\} .
$$

Set $g_{r}=\Phi_{r} \circ f$. We shall give the properties of $\phi(r)$, which was first considered by H. Grötzsch for $f K$-quasiconformal (see [5] and [2]). However, it is not obvious from conditions (a) and (b) that the sense-preserving mapping 
$f$ in Theorem 2.1 is $K$-quasiconformal. Following Grötzsch's idea, we prove Lemma 2.4 directly by extremal length methods.

Lemma 2.4. If $f$ satisfies condition (a), then $\phi(r) / r^{1 / K}$ is an increasing function on $(0,1]$, and $\phi(r) / r^{1 / K} \leq 1$.

Proof. Assume that $0<r_{1}<r_{2} \leq 1$. Then $g_{r_{1}}(z)$ maps $\left\{z\left|r_{1} \leq\right| z \mid \leq 1\right\}$ onto $\left\{\zeta\left|\phi\left(r_{1}\right) \leq\right| \zeta \mid \leq 1\right\}$. The definition of moduli of ring domains together with Proposition 1.2 yields

$$
\begin{aligned}
\bmod g_{r_{1}}\left\{z\left|r_{1} \leq\right| z \mid\right. & \left.\leq r_{2}\right\}+\bmod g_{r_{1}}\left\{z\left|r_{2} \leq\right| z \mid \leq 1\right\} \\
& \leq \bmod g_{r_{1}}\left\{z\left|r_{1} \leq\right| z \mid \leq 1\right\}=\frac{1}{2 \pi} \log \frac{1}{\phi\left(r_{1}\right)} .
\end{aligned}
$$

From conformal invariance of extremal length, it follows that

$$
\begin{aligned}
\bmod g_{r_{1}}\left\{z\left|r_{2} \leq\right| z \mid \leq 1\right\} & =\bmod f\left\{z\left|r_{2} \leq\right| z \mid \leq 1\right\} \\
& =\bmod g_{r_{2}}\left\{z\left|r_{2} \leq\right| z \mid \leq 1\right\}=\frac{1}{2 \pi} \log \frac{1}{\phi\left(r_{2}\right)} .
\end{aligned}
$$

Substituting (2.8) into (2.7), we have

$$
\bmod g_{r_{1}}\left\{z\left|r_{1} \leq\right| z \mid \leq r_{2}\right\} \leq \frac{1}{2 \pi} \log \frac{\phi\left(r_{2}\right)}{\phi\left(r_{1}\right)} .
$$

On the other hand, from condition (a) it follows that

$$
\bmod g_{r_{1}}\left\{z\left|r_{1} \leq\right| z \mid \leq r_{2}\right\}=\bmod f\left\{z\left|r_{1} \leq\right| z \mid \leq r_{2}\right\} \geq \frac{1}{2 \pi K} \log \frac{r_{2}}{r_{1}} .
$$

Combining (2.9) with (2.10), we obtain

$$
\left(\frac{r_{2}}{r_{1}}\right)^{1 / K} \leq \frac{\phi\left(r_{2}\right)}{\phi\left(r_{1}\right)}, \quad \text { that is, } \quad \frac{\phi\left(r_{1}\right)}{r_{1}^{1 / K}} \leq \frac{\phi\left(r_{2}\right)}{r_{2}^{1 / K}} .
$$

So, the monotonicity of $\phi(r) / r^{1 / K}$ is proved. From the above inequality, by letting $r_{2}=1$, we get $\phi(r) / r^{1 / K} \leq 1$ for $r \in(0,1]$. This completes the proof of the lemma.

LEMmA 2.5. If $f$ satisfies condition (b), then

$$
\lim _{r \rightarrow 0} \frac{\phi(r)}{r^{1 / K}}=1 \text {. }
$$

Proof. For any $\varepsilon>0$, there exists $\delta>0$ such that whenever $r<\delta$,

$$
1-\varepsilon<\frac{\left|f\left(r e^{i \theta}\right)\right|}{r^{1 / K}}<1+\varepsilon
$$

Therefore,

$$
\left\{w\left|(1+\varepsilon) r^{1 / K}<\right| w \mid \leq 1\right\} \subset f(R(r)) \subset\left\{w\left|(1-\varepsilon) r^{1 / K}<\right| w \mid \leq 1\right\} .
$$


By Proposition 1.1,

$$
\frac{1}{2 \pi} \log \frac{1}{(1+\varepsilon) r^{1 / K}} \leq \bmod f(R(r)) \leq \frac{1}{2 \pi} \log \frac{1}{(1-\varepsilon) r^{1 / K}}
$$

Therefore,

$$
\frac{1}{2 \pi} \log \frac{1}{(1+\varepsilon) r^{1 / K}} \leq \frac{1}{2 \pi} \log \frac{1}{\phi(r)} \leq \frac{1}{2 \pi} \log \frac{1}{(1-\varepsilon) r^{1 / K}}
$$

That is,

$$
1-\varepsilon \leq \frac{\phi(r)}{r^{1 / K}} \leq 1+\varepsilon .
$$

This shows $\lim _{r \rightarrow 0} \phi(r) / r^{1 / K}=1$.

Proof of Theorem 2.1. From Lemmas 2.4 and 2.5, we obtain

$$
\phi(r)=r^{1 / K}, \quad 0 \leq r \leq 1 .
$$

This means

$$
\left|g_{r}(z)\right|=|z|^{1 / K} \quad(r \leq|z| \leq 1)
$$

Taking $r=1 / n$, we write

$$
g_{n}(z)=|z|^{1 / K} e^{i h_{n}(\theta)} \quad(1 / n \leq|z| \leq 1),
$$

where $\theta=\arg z$ and $h_{n}$ is a homeomorphism from $[0,2 \pi]$ onto $\left[\theta_{n}, \theta_{n}+2 \pi\right]$ $\left(0 \leq \theta_{n}<2 \pi\right)$. According to Lemma 2.3, $f$ is absolutely continuous on $\{|z|=r\}$, and so is $g_{n}$. Therefore, $h_{n}^{\prime}(\theta)$ exists almost everywhere and is integrable on $[0,2 \pi]$, and

$$
\int_{0}^{2 \pi} h_{n}^{\prime}(\theta) d \theta=2 \pi
$$

Direct computation on generalized derivatives of $g_{n}(z)$ in (2.11) shows

$$
\begin{aligned}
& \partial_{z} g_{n}(z)=\frac{z}{2|z|^{2+1 / K}} e^{-i h_{n}(\theta)}\left(\frac{1}{K}+h_{n}^{\prime}(\theta)\right), \\
& \partial_{\bar{z}} g_{n}(z)=\frac{\bar{z}}{2|z|^{2+1 / K}} e^{-i h_{n}(\theta)}\left(\frac{1}{K}-h_{n}^{\prime}(\theta)\right) .
\end{aligned}
$$

Since $g_{n}$ is sense-preserving,

$$
\left|\partial_{z} g_{n}(z)\right|>\left|\partial_{\bar{z}} g_{n}(z)\right| \text {. }
$$

Therefore, from (2.13) and (2.14) we infer that $h_{n}^{\prime}(\theta)>0$. Set

$$
P=\{z|r \leq| z \mid \leq r+d r, \theta \leq \arg z \leq \theta+d \theta\}
$$

Then

$$
g_{n}(P)=\left\{\zeta\left|r^{1 / K} \leq\right| \zeta \mid \leq(r+d r)^{1 / K}, h_{n}(\theta) \leq \arg \zeta \leq h_{n}(\theta)+h_{n}^{\prime}(\theta) d \theta\right\} .
$$


By conformal invariance of extremal length and condition (a), we have

$$
\bmod g_{n}(P) \geq \frac{1}{K} \bmod P \text {. }
$$

From the above inequality, it follows that $h_{n}^{\prime}(\theta) \leq 1$. Thus,

$$
0<h_{n}^{\prime}(\theta) \leq 1 \text {. }
$$

From (2.12) and (2.15), we obtain $h_{n}^{\prime}(\theta)=1$ a.e. on $[0,2 \pi)$. Therefore, $h_{n}(\theta)=\theta_{n}+\theta$, and we can rewrite $(2.11)$ as follows:

$$
g_{n}(z)=z|z|^{1 / K-1} e^{i \theta_{n}} \quad(1 / n \leq|z| \leq 1) .
$$

Since $\theta_{n} \in[0,2 \pi)$, there exists a subsequence $\left\{\theta_{n_{k}}\right\}$ such that $\lim _{k \rightarrow \infty} \theta_{n_{k}}$ $=\theta_{0}$. Therefore,

$$
\lim _{k \rightarrow \infty} g_{n_{k}}(z)=g(z)=z|z|^{1 / K-1} e^{i \theta_{0}} \quad \text { (locally uniformly in } \Delta-\{0\} \text { ). }
$$

So,

$$
\begin{aligned}
\lim _{k \rightarrow \infty} \Phi_{n_{k}}(w) & =\lim _{k \rightarrow \infty} g_{n_{k}} \circ f^{-1}(w) \\
& \left.=g \circ f^{-1}(w):=\Phi(w) \quad \text { (locally uniformly in } \Delta-\{0\}\right) .
\end{aligned}
$$

From compactness of the family of analytic functions, $\Phi$ is analytic in $\Delta-\{0\}$. Observing that $\lim _{w \rightarrow 0} \Phi(w)=0$, we conclude that the origin is a removable singular point and hence $\Phi$ is analytic in the unit disk. Since

$$
\lim _{w \rightarrow 0} \frac{|\Phi(w)|}{|w|}=\lim _{z \rightarrow 0} \frac{\Phi \circ f(z)}{f(z)}=\lim _{z \rightarrow 0} \frac{|z|^{1 / K}}{|f(z)|}=1,
$$

by the Schwarz lemma we have $\Phi(w)=\lambda w$. Thus, $f(z)=\lambda z|z|^{1 / K-1}$ where $|\lambda|=1$.

COROLlary 2.6. If $f$ is $K$-quasiconformal mapping of $\Delta$ onto itself which satisfies $f(0)=0$ and

$$
\lim _{z \rightarrow 0} \frac{|f(z)|}{|z|^{K}}=1,
$$

then $f(z)=\lambda z|z|^{K-1}$.

Problem 2.7. If we replace conditions (a) and (b) by

$$
\begin{gathered}
\bmod f(A) \leq K \bmod A \quad \text { for all } A \subset \Delta, \\
\lim _{z \rightarrow 0} \frac{|f(z)|}{|z|^{K}}=1,
\end{gathered}
$$

is it true that $f(z)=\lambda z|z|^{K-1}$ ? 


\section{Affine mappings on rectangles}

Lemma 3.1 ([1]). Let $R=\{z \mid 0 \leq x \leq a, 0 \leq y \leq 1\}$. Let $C$ be a curve in $R$ that connects the a-sides of $R$ and hence divides $R$ into two parts $R_{1}$ and $R_{2}$. Then $R_{1}$ and $R_{2}$ are rectangles if and only if

$$
\bmod R_{1}+\bmod R_{2}=\bmod R=a .
$$

LEMma 3.2. Let $f$ be a sense-preserving homeomorphism which maps $\left\{z\left|r_{0} \leq\right| z \mid \leq 1\right\}$ onto $\left\{w\left|r_{0}^{1 / K} \leq\right| w \mid \leq 1\right\}$. If

$$
\frac{1}{K} \bmod A \leq \bmod f(A)
$$

for all $A \subset\left\{z\left|r_{0} \leq\right| z \mid \leq 1\right\}$ appearing in (1.6) and (1.8), then

$$
f(z)=\lambda z|z|^{1 / K-1} \quad(|\lambda|=1) .
$$

Proof. Extend $f$ by setting

$$
f(z)=r_{0}^{2 n / K} / f\left(r_{0}^{2 n} / \bar{z}\right), \quad r_{0}^{n+1} \leq|z| \leq r_{0}^{n}, n=1,2, \ldots
$$

Then the extended $f$ is a sense-preserving homeomorphism of $\Delta$ onto itself with $f(0)=0$. Furthermore, it is not difficult to prove that the extended $f$ satisfies (a) and (b). Hence, by Theorem 2.1,

$$
f(z)=\lambda z|z|^{1 / K-1} \text {. }
$$

TheOREM 3.3. Assume that $f$ is a sense-preserving homeomorphism which maps the rectangle $R=\{z \mid 0 \leq x \leq a, 0 \leq y \leq 1\}$ onto $f(R)=$ $\left\{w \mid 0 \leq u \leq a^{\prime}, 0 \leq v \leq 1\right\}$. If for every horizontal subrectangle $R_{h}(\subset R)$,

$$
\frac{1}{K} \bmod R_{h} \leq \bmod f\left(R_{h}\right)
$$

then

$$
f(z)=\frac{1}{K} x+i y
$$

where $K=\bmod R / \bmod f(R)=a / a^{\prime}$.

Proof. Set $R_{1}=\left\{z \mid 0 \leq x \leq a_{1}, 0 \leq y \leq 1\right\}, R_{2}=\left\{z \mid a_{1} \leq x \leq a\right.$, $0 \leq y \leq 1\}$. By (3.1), we have

$$
\frac{1}{K} \bmod R_{1} \leq \bmod f\left(R_{1}\right), \quad \frac{1}{K} \bmod R_{2} \leq \bmod f\left(R_{2}\right) .
$$

Therefore,

$$
\frac{1}{K} \bmod R=\frac{1}{K}\left(\bmod R_{1}+\bmod R_{2}\right) \leq \bmod f\left(R_{1}\right)+\bmod f\left(R_{2}\right) .
$$

On the other hand, by Proposition 1.2,

$$
\bmod f\left(R_{1}\right)+\bmod f\left(R_{2}\right) \leq \bmod f(R)=\frac{1}{K} \bmod R .
$$


Combining (3.3) with (3.4), we have

$$
\bmod f\left(R_{1}\right)+\bmod f\left(R_{2}\right)=\bmod f(R) .
$$

By Lemma 3.1, $f\left(R_{1}\right)$ and $f\left(R_{2}\right)$ are rectangles. That is,

$$
f(x)=f(x+i), \quad 0 \leq x \leq a .
$$

It follows from (3.5) that $f$ can be extended to the strip

$$
S=\{z \mid 0 \leq x \leq a\} .
$$

By the extremal length method, it is not difficult to prove that for the extended function $f$, which maps $S$ onto another strip

$$
S^{\prime}=\left\{w \mid 0 \leq u \leq a^{\prime}\right\},
$$

inequality (3.1) still holds for any $R_{h} \subset S$. Set

$$
G=\left\{\zeta\left|e^{-a} \leq\right| \zeta \mid \leq 1\right\}, \quad G^{\prime}=\left\{\eta\left|e^{-a^{\prime}} \leq\right| \eta \mid \leq 1\right\} .
$$

Then $\left\{S, e^{-a+z}\right\},\left\{S^{\prime}, e^{-a^{\prime}+w}\right\}$ are universal covering surfaces of $G$ and $G^{\prime}$ respectively.

The projective mapping of the function $f$,

$$
g(\zeta)=e^{-a^{\prime}+f(a+\log \zeta)},
$$

is a sense-preserving homeomorphism and satisfies inequality (3.0). In view of Lemma 3.2, we have

$$
g(\zeta)=\lambda \zeta|\zeta|^{1 / K-1} \quad(|\lambda|=1)
$$

Combining (3.6) with (3.7) and observing that $f(0)=0$, we obtain

$$
f(z)=\frac{1}{K} x+i y \text {. }
$$

This ends the proof of the theorem.

The above theorem yields immediately

COROLlary 3.4. Let $f$ be a sense-preserving homeomorphism which maps a rectangle $R$ onto a quadrilateral $Q$ and satisfies the following two conditions:

(c) $K^{-1} \bmod R_{h} \leq \bmod f\left(R_{h}\right)$ for every horizontal subrectangle $R_{h} \subset R$,

(d) $K^{-1} \bmod R=\bmod Q$.

Then $f$ is $K$-quasiconformal and is a Teichmüller mapping.

REMARK 3.5. It might seem that Corollary 3.4 should have a symmetric form, with (c) and (d) replaced respectively by

$$
\begin{aligned}
\bmod R_{h} & \leq K \bmod f\left(R_{h}\right), \\
\bmod R & =K \bmod f\left(R_{h}\right) .
\end{aligned}
$$


But unfortunately, this is not the case. In fact, we can find a counterexample in [3], where $f$ is $\left(K+\sqrt{K^{2}-1}\right)$-quasiconformal.

4. Geometric characterization for Teichmüller mappings. Let $S_{1}$ and $S_{2}$ be two Riemann surfaces. Let $\omega$ be a holomorphic quadratic differential on $S_{1}$. A quasiconformal mapping $f$ from $S_{1}$ onto $S_{2}$ is called a Teichmüller mapping if its Beltrami differential $\mu_{f} d \bar{z} / d z$ equals $k \bar{\omega} / \omega$. In this section, we want to characterize Teichmüller mappings by a local property.

Let $D_{1}$ and $D_{2}$ be simply connected domains of hyperbolic type. From the Riemann mapping theorem, there exist conformal mappings $\Phi_{1}$ and $\Phi_{2}$ which map $D_{1}$ and $D_{2}$ respectively onto $\Delta$.

TheOREM 4.1. Suppose that $f$ is a $K$-quasiconformal mapping from $D_{1}$ onto $D_{2}$. To $z_{0} \in D_{1}$, there correspond conformal mappings $\Phi_{1}$ and $\Phi_{2}$ as above with $\Phi_{1}\left(z_{0}\right)=0$ and $\Phi_{2}\left(f\left(z_{0}\right)\right)=0$. If either

$$
\lim _{z \rightarrow z_{0}} \frac{\left|f(z)-f\left(z_{0}\right)\right|}{\left|z-z_{0}\right|^{1 / K}}=\frac{\left|\Phi_{1}^{\prime}\left(z_{0}\right)\right|^{1 / K}}{\left|\Phi_{2}^{\prime}\left(f\left(z_{0}\right)\right)\right|}
$$

or

$$
\lim _{z \rightarrow z_{0}} \frac{\left|f(z)-f\left(z_{0}\right)\right|}{\left|z-z_{0}\right|^{K}}=\frac{\left|\Phi_{1}^{\prime}\left(z_{0}\right)\right|^{K}}{\left|\Phi_{2}^{\prime}\left(f\left(z_{0}\right)\right)\right|},
$$

then $f$ is a Teichmüller mapping.

Proof. Let $w=f(z), \zeta=\Phi_{1}(z), \eta=\Phi_{2}(w)$. Set $g(\zeta)=\Phi_{2} \circ f \circ \Phi_{1}^{-1}(\zeta)$. From (4.1) and (4.2), it is not difficult to prove that

$$
\text { either } \lim _{\zeta \rightarrow 0} \frac{|g(\zeta)|}{|\zeta|^{1 / K}}=1 \quad \text { or } \quad \lim _{\zeta \rightarrow 0} \frac{|g(\zeta)|}{|\zeta|^{K}}=1 .
$$

Therefore, by Theorem 2.1 and Corollary 2.6, $g(\zeta)$ is an affine mapping. Thus, $f$ is a Teichmüller mapping.

The above theorem yields immediately

COROLlary 4.2. Suppose that $f$ is a $K$-quasiconformal mapping of $\Delta$ onto itself. If either

$$
\lim _{z \rightarrow z_{0}} \frac{\left|f(z)-f\left(z_{0}\right)\right|}{\left|z-z_{0}\right|^{1 / K}}=\frac{1-\left|f\left(z_{0}\right)\right|^{2}}{\left(1-\left|z_{0}\right|^{2}\right)^{1 / K}}
$$

or

$$
\lim _{z \rightarrow z_{0}} \frac{\left|f(z)-f\left(z_{0}\right)\right|}{\left|z-z_{0}\right|^{K}}=\frac{1-\left|f\left(z_{0}\right)\right|^{2}}{\left(1-\left|z_{0}\right|^{2}\right)^{K}}
$$

then $f$ is a Teichmüller mapping.

THEOREM 4.3. Let $\left(\Delta, \pi_{1}\right)$ and $\left(\Delta, \pi_{2}\right)$ be universal covering surfaces of $S_{1}$ and $S_{2}$ respectively. Let $\tilde{f}: \Delta \rightarrow \Delta$ be a lift of a $K$-quasiconformal 
mapping $f$ of $S_{1}$ onto $S_{2}$. If for a neighbourhood $V$ of a lift of $f\left(p_{0}\right)$, either

$$
\begin{aligned}
\lim _{p \rightarrow p_{0}} \frac{\left|\left(\left.\pi_{2}\right|_{V}\right)^{-1} \circ f(p)-\left(\left.\pi_{2}\right|_{V}\right)^{-1} \circ f\left(p_{0}\right)\right|}{\left|\left(\left.\pi_{1}\right|_{\tilde{f}^{-1}(V)}\right)^{-1}(p)-\left(\left.\pi_{1}\right|_{\widetilde{f}^{-1}(V)}\right)^{-1}\left(p_{0}\right)\right|^{1 / K}} & \\
& =\frac{1-\left|\left(\left.\pi_{2}\right|_{V}\right)^{-1} \circ f\left(p_{0}\right)\right|^{2}}{\left(1-\left|\left(\left.\pi_{1}\right|_{\widetilde{f}^{-1}(V)}\right)^{-1}\left(p_{0}\right)\right|^{2}\right)^{1 / K}}
\end{aligned}
$$

or

$$
\lim _{p \rightarrow p_{0}} \frac{\left|\left(\pi_{2} \mid V\right)^{-1} \circ f(p)-\left(\pi_{2} \mid V\right)^{-1} \circ f\left(p_{0}\right)\right|}{\left|\left(\left.\pi_{1}\right|_{\tilde{f}^{-1}(V)}\right)^{-1}(p)-\left(\left.\pi_{1}\right|_{\tilde{f}^{-1}(V)}\right)^{-1}\left(p_{0}\right)\right|^{K}}=\frac{1-\left|\left(\pi_{2} \mid V\right)^{-1} \circ f\left(p_{0}\right)\right|^{2}}{\left(1-\left|\left(\left.\pi_{1}\right|_{\tilde{f}^{-1}(V)}\right)^{-1}\left(p_{0}\right)\right|^{2}\right)^{K}}
$$

then $f$ is a Teichmüller mapping.

Proof. By hypothesis, $\tilde{f}$ is a $K$-quasiconformal mapping of $\Delta$ onto itself and satisfies (4.3) or (4.4). In view of Corollary 4.2, $\widetilde{f}$ is a Teichmüller mapping. Hence so is $f$.

\section{References}

[1] L. V. Ahlfors, Lectures on Quasiconformal Mappings, Van Nostrand, Princeton, 1966.

[2] P. P. Belinskiı̌, General Properties of Quasiconformal Mappings, Nauka, Novosibirsk, 1974 (in Russian).

[3] A. C. Cabiria, On the geometric definition of quasiconformality, Ann. Polon. Math. 39 (1981), 31-36.

[4] F. W. Gehring and J. Väisälä, On the geometric definition for quasiconformal mappings, Comment. Math. Helv. 36 (1962), 19-32.

[5] H. Grötzsch, Über die Verzerrung bei schlichten nichtkonformen Abbildungen und über eine damit zusammenhängende Erweiterung des Picardschen Satzes, Sitzungsber. Sächs. Akad. Wiss. Phys. Kl. Leipzig, 1928.

[6] R. Kühnau, Herbert Grötzsch zum Gedächtnis, Jahresber. Deutsch. Math.-Verein. 99 (1997), 122-145.

[7] O. Lehto and K. I. Virtanen, Quasiconformal Mappings in the Plane, Springer, Berlin, 1973.

[8] H. Zhu, Z. Zhou and C. He, The characterization of Grötzsch's problem in a domain, J. Fudan Univ. 38 (1999), 205-207 (in Chinese).

Department of Mathematics

XiXi campus

Zhejiang University

Hangzhou, Zhejiang, 310028

P.R. China

E-mail: zgchen@zju.edu.cn 